\title{
The Sleep-Promoting and Hypothermic Effects of Glycine are Mediated by NMDA Receptors in the Suprachiasmatic Nucleus
}

\author{
Nobuhiro Kawai ${ }^{1,4}$, Noriaki Sakai ${ }^{2,4}$, Masashi Okuro ${ }^{2,3}$, Sachie Karakawa', Yosuke Tsuneyoshi', \\ Noriko Kawasaki', Tomoko Takeda', Makoto Bannai*,' and Seiji Nishino ${ }^{2}$ \\ IInstitute for Innovation, Ajinomoto Co., Inc., Kawasaki, Japan; ${ }^{2}$ Sleep and Circadian Neurobiology laboratory, Stanford University School of \\ Medicine, Stanford, CA, USA; ${ }^{3}$ Department of Geriatric Medicine, Kanazawa Medical University, Kanazawa, Japan
}

\begin{abstract}
The use of glycine as a therapeutic option for improving sleep quality is a novel and safe approach. However, despite clinical evidence of its efficacy, the details of its mechanism remain poorly understood. In this study, we investigated the site of action and sleep-promoting mechanisms of glycine in rats. In acute sleep disturbance, oral administration of glycine-induced non-rapid eye movement (REM) sleep and shortened NREM sleep latency with a simultaneous decrease in core temperature. Oral and intracerebroventricular injection of glycine elevated cutaneous blood flow (CBF) at the plantar surface in a dose-dependent manner, resulting in heat loss. Pretreatment with N-methyl-D-aspartate (NMDA) receptor antagonists AP5 and CGP78608 but not the glycine receptor antagonist strychnine inhibited the CBF increase caused by glycine injection into the brain. Induction of c-Fos expression was observed in the hypothalamic nuclei, including the medial preoptic area (MPO) and the suprachiasmatic nucleus (SCN) shell after glycine administration. Bilateral microinjection of glycine into the SCN elevated CBF in a dose-dependent manner, whereas no effect was observed when glycine was injected into the MPO and dorsal subparaventricular zone. In addition, microinjection of D-serine into the SCN also increased CBF, whereas these effects were blocked in the presence of L-701324. SCN ablation completely abolished the sleep-promoting and hypothermic effects of glycine. These data suggest that exogenous glycine promotes sleep via peripheral vasodilatation through the activation of NMDA receptors in the SCN shell.
\end{abstract}

Neuropsychopharmacology (20I5) 40, I405-14I6; doi:I0.1038/npp.20 I4.326; published online I4 January 2015

\section{INTRODUCTION}

Glycine, a non-essential amino acid, has two distinct properties as a neurotransmitter: it operates through two different receptors and engages in several functions in the central nervous system (CNS). Glycine has long been known as an inhibitory neurotransmitter in the brainstem and spinal cord (Curtis et al, 1968; Werman et al, 1968). In these areas, the activation of glycinergic premotoneurons triggers muscle atonia during rapid eye movement (REM) sleep (Chase et al, 1989; Holstege and Bongers, 1991; Morales et al, 2006; Soja et al, 1991). By contrast, glycine acts allosterically as an excitatory modulator of the N-methyl-D-aspartate (NMDA) subtype of ionotropic glutamate receptors (Johnson and Ascher, 1987; Kleckner and Dingledine, 1988).

Although sufficient amounts of glycine are synthesized de novo or are taken up from the diet daily (Reeds, 2000),

\footnotetext{
*Correspondence: Dr M Bannai, Institute for Innovation, Ajinomoto Co., Inc., Kawasaki 210 868I, Kanagawa, Japan, Tel: + I 8144244 8678, Fax: + I 8144244 1046, E-mail: makoto_bannai@ajinomoto.com ${ }^{4}$ These authors contributed equally to the work.

Received 15 May 20I4; revised 17 November 2014; accepted I December 2014; accepted article preview online 23 December 2014
}

exogenous glycine passively diffuses across the blood-brain barrier and modulates neurotransmission in the CNS (Kawai et al, 2011; Peterson, 1994; Toth and Lajtha, 1986). Because glutamatergic neurotransmission has been implicated in schizophrenia pathophysiology, oral administration of high-dose glycine-a mean dose of $\sim 60 \mathrm{~g} /$ day-has been reported to significantly improve cognition and reduce the negative symptoms of schizophrenia (Coyle and Tsai, 2004; Heresco-Levy et al, 1999). Conversely, low-dose glycine at a dose of $3 \mathrm{~g} /$ day before bedtime subjectively improves sleep quality and reduces sleepiness and fatigue during the day in individuals with insomniac tendencies or restricted sleep time (Bannai et al, 2012; Inagawa et al, 2006). A polysomnographic study also revealed that the same dose of glycine stabilizes sleep state and shortens the latency to slow-wave sleep, with no alterations in sleep architecture (Yamadera et al, 2007). A recent study reported that the intraperitoneal injection of glycine inhibits the activity of orexin neurons, which are critical for arousal, energy homeostasis, and reward-seeking (Sakurai et al, 2010) and induces non-REM (NREM) sleep with fragmented vigilance states in mice (Hondo et al, 2011). However, Karnani et al (2011a) showed that orexin neurons are stimulated by nutritionally relevant amino acid mixtures, 
and of the amino acids tested, glycine had the highest potency. Thus, the site of action and the sleep-promoting mechanism of glycine have remained controversial.

A temporal relationship between the timing of sleep and core body temperature $(\mathrm{Tb})$ rhythm has been welldocumented in humans (Barrett et al, 1993; Campbell and Broughton, 1994; Czeisler et al, 1980; Dijk and von Schantz, 2005; Zulley et al, 1981) and rats (Alfoldi et al, 1990). Some hypnotics, such as benzodiazepines, zolpidem, and ramelteon, induce hypothermia within a physiological range (Elliot and White, 2001; Markwald et al, 2010). In some human studies, exogenous melatonin has been shown to induce sleep and lower Tb (Lavie, 1997; Stone et al, 2000; Zhdanova et al, 1995). Similarly, glycine administration before bedtime decreases $\mathrm{Tb}$ in human subjects (Nagao et al, 2007).

In the present study, we aimed to elucidate the mechanisms involved in the sleep-promoting and hypothermic effects of glycine in rats. We first demonstrated that glycine administration alleviates acute sleep disturbance. Laser-Doppler imaging was used to assess peripheral blood flow both before and after glycine injection into the stomach or brain. The site of glycine action was determined by c-Fos immunohistochemical analysis and microinjection into the hypothalamic nuclei in the absence and presence of specific NMDA receptor antagonists. Suprachiasmatic nucleus (SCN) ablation completely abolished the sleep-promoting and hypothermic effects of glycine. Our results consistently suggest that exogenous glycine promotes sleep via peripheral vasodilatation through the activation of NMDA receptors in the SCN.

\section{MATERIALS AND METHODS}

\section{Animals}

Male Sprague-Dawley rats were purchased, weighing 250$300 \mathrm{~g}$, from Charles River Laboratories (Hollister, CA) for use in the sleep study (electroencephalogram/electromyogram, EEG/EMG recording). Male Wistar rats were purchased, weighing 250-300 g, from Charles River Japan (Yokohama, Japan) and were also used for the other studies. The animals were housed individually in plastic cages and maintained under standard conditions in a 12-h light-dark cycle with access to food and water ad libitum. All procedures were performed in accordance with the guidelines of National Institutes of Health and the Animal Care Committee of Ajinomoto.

\section{Sleep Analysis}

Implant surgery, EEG/EMG monitoring, and EEG data acquisition were performed as previously described (Fujiki et al, 2009). To evaluate the effects of glycine administration on locomotor activity and $\mathrm{Tb}$, a telemetry device (G2 E-Mitter, Mini Mitter, OR; or TA-F40, DSI, St. Paul, MN) was implanted in the abdominal cavity of each rat.

After 2 weeks of recovery, the rats were acclimatized to the recording cable for at least 10 days in the individual recording cage. On the day of recording, $2 \mathrm{~g} / \mathrm{kg}$ glycine (Sigma, St Louis, MO) or water (vehicle) was orally administered $2 \mathrm{~h}$ after the onset of the light period (ZT2), and the rats were placed into a new, clean cage, which induces stress and acute sleep disturbance (Cano et al, 2008; Oka et al, 2001). The dose administered is sufficient to cross the blood-brain barrier (Kawai et al, 2011). Sleep and telemetry data were recorded for $6 \mathrm{~h}$.

\section{Core Body Temperature}

Core body temperature was measured by the methods described above. $2 \mathrm{~g} / \mathrm{kg}$ glycine or water (vehicle) was orally administered $4 \mathrm{~h}$ after the onset of the dark period (ZT16) in a habituated cage without sleep recording (Figure $2 \mathrm{a}$ and $\mathrm{b}$ ).

\section{Laser-Doppler Imaging of Rat Skin Blood Flow}

The units of laser-Doppler perfusion were used as an indicator of cutaneous blood flow (CBF) because they closely correlate with CBF (Johnson et al, 1984). The rats were anesthetized by isoflurane (maintained with $1.5 \%$, $1.0 \mathrm{ml} / \mathrm{min}$ ) at ZT3-4 and placed on a heating pad (CMA150; CMA/Microdialysis, Stockholm, Sweden) to maintain a rectal temperature between 37.0 and $37.5^{\circ} \mathrm{C}$ during the procedure. To measure CBF, the rats were fixed in a prone position, and the laser-Doppler perfusion imager (Periscan PIMII; Perimed AB, Stockholm, Sweden) was set to a position $\sim 15 \mathrm{~cm}$ above the left plantar surface, upon which, the laser beam was directed. The continuous perfusion units of the plantar surface were recorded every 3 min during the experimental periods. The mean values of the perfusion units of the whole-plantar surface were automatically calculated as time-point data. After stabilization of the perfusion units of the plantar surface, 26 rats were administered two doses of glycine ( 1 or $2 \mathrm{~g} / \mathrm{kg}$ ) or water (vehicle) via a gavage needle into the stomach.

For intracerebroventricular (ICV) or intracerebral injections, 42 rats were surgically implanted with guide cannula (26 gauge; Plastics One, Roanoke,VA) stereotaxically positioned $1 \mathrm{~mm}$ above the lateral ventricle or each hypothalamic area (MPO; dorsal subparaventricular zone, dSPZ; $\mathrm{SCN})$ under pentobarbital anesthesia $(50 \mathrm{mg} / \mathrm{kg}$, ip). The stereotaxic coordinates were anterior-posterior (AP) +0.8 (in $\mathrm{mm}$, from bregma), medial-lateral (ML) +1.6 , dorsalventral (DV) -4.0 (from the skull surface) for the lateral ventricle; $\mathrm{AP}-0.7, \mathrm{ML} \pm 0.8$, DV -8.8 for $\mathrm{MPO}$; $\mathrm{AP}-1.8$, $\mathrm{ML} \pm 0.5, \mathrm{DV}-8.0$ for dSPZ; AP $-0.7, \mathrm{ML} \pm 1.0 \mathrm{DV}-9.4$ with $5^{\circ}$ angle for SCN (bilateral), according to the atlas of Paxinos and Watson (2004). After recovery from surgery, 13,65 , or $130 \mathrm{nmol}$ of glycine, $1000 \mathrm{nmol}$ of D-serine, 130 or $1000 \mathrm{nmol}$ of L-serine, or artificial cerebrospinal fluid (CMA/Microdialysis) was administered into the lateral ventricle or hypothalamic area through an inserted guide cannulae, and the change in the perfusion units was evaluated. To antagonize either glycine receptors or NMDA receptors, antagonists were applied through a cannulae 15 min before administration of vehicle, glycine, or D-serine (Sigma). The glycine receptor antagonist strychnine (30 nmol; Sigma), selective NMDA receptor antagonist DLAP5 (5 nmol; Sigma), glycine site-specific NMDA receptor antagonist CGP78608 (20 pmol; Tocris, Bristol, UK) and strychnine-insensitive NMDA receptor glycine site antagonist L-701324 (1 nmol; Tocris) were used to antagonize 
glycine or NMDA receptors. The injection volume was $1 \mu \mathrm{l}$ at a rate of $1 \mu \mathrm{l} / \mathrm{min}$.

\section{c-Fos Immunohistochemistry}

The rats were orally administered glycine $(2 \mathrm{~g} / \mathrm{kg}, n=5)$ or vehicle $(n=5)$ at ZT16 and were killed in the dark $30 \mathrm{~min}$ after administration. Whole brains were removed, postfixed overnight at $4{ }^{\circ} \mathrm{C}$ in $4 \%$ paraformaldehyde, and then cryoprotected in $30 \%$ sucrose in PBS at $4{ }^{\circ} \mathrm{C}$ for $48 \mathrm{~h}$. Cryoprotected whole brains were sliced coronally into $50-\mu \mathrm{m}$ sections with a cryostat (Leica CM3050S, Leica Microsystems, Wetzlar, Germany). The sections were incubated with $0.3 \% \mathrm{H}_{2} \mathrm{O}_{2}$ for $30 \mathrm{~min}$ at room temperature to block endogenous peroxidase activities, followed by three washes in $0.3 \%$ Triton PBS, then incubated overnight at $4{ }^{\circ} \mathrm{C}$ in $0.3 \%$ Triton PBS with $3 \%$ normal rabbit serum containing goat anti-c-Fos antibody (1:2000, sc-52-g, Santa Cruz Biotechnology, CA). After rinsing, the sections were incubated for $1 \mathrm{~h}$ in $0.3 \%$ Triton PBS with 3\% normal rabbit serum containing biotinylated rabbit anti-goat IgG, and incubated for $(1: 200$, BA-5000, Vector Laboratories, CA) followed by incubation with avidin-biotin complex $(1: 500$, PK-6105, Vector Laboratories). Staining for c-Fos immunoreactivity was visualized with nickel-enhanced 3,3'-diaminobenzidine in Tris-HCl (0.05 M, pH 7.6, SK-4100, Vector Laboratories), yielding a black reaction product that was produced by a modified glucose oxidase protocol that generates hydrogen peroxide in the incubation solution. The sections were mounted on gelatin-coated slides, dehydrated, and coverslipped. A single observer, blinded for the treatment, counted c-Fos immunoreactive cells manually using ImageJ (NIH software). The standard areas of analysis were determined in accordance with previously defined landmarks (Swanson, 2004). The areas were as follows: the medial preoptic area (mPO, $400 \times 400 \mu \mathrm{m})$; the periventricular/paraventricular hypothalamic nucleus $(\mathrm{Pe} / \mathrm{Pa}, 400 \times 400 \mu \mathrm{m})$; and the $\mathrm{SCN}(300-\mu \mathrm{m}$ circle). The SCN subdivisions were defined as previously described (Sumova et al, 1998). For each rat, the counts were averaged to derive the mean number of c-Fos-reactive cells per unilateral structure.

\section{SCN Lesions}

Adult male rats were randomly assigned to the sham $(n=6)$ and the lesioned $(n=14)$ group. The rats were anesthetized with $3 \%$ isoflurane and placed in a stereotaxic instrument. Bilateral radio frequency lesions of the $\mathrm{SCN}$ were created by raising the probe tip temperature to $63^{\circ} \mathrm{C}$ at each lesion site. To create a lesion, the tip of the probe was placed $0.8 \mathrm{~mm}$ caudal to the bregma, $0.3 \mathrm{~mm}$ bilateral to the sagittal sinus, and $8.8 \mathrm{~mm}$ ventral to the dura. The probe was lowered $0.5 \mathrm{~mm}$ above the SCN but not heated to make sham lesions in the sham group. After surgery, successful lesioning of the SCN was confirmed by the loss of circadian rhythms in $\mathrm{Tb}$ during constant dark conditions for 3 weeks. Behavioral arrhythmicity was tested by a $\chi^{2}$ periodogram using the $\mathrm{Tb}$ data. The successful SCN-lesioned (SCNx, $n=11)$ and sham $(n=6)$ groups were implanted with EEG/EMG electrodes and used in the sleep study. The sham and SCNx groups received vehicle or glycine $(2 \mathrm{~g} / \mathrm{kg})$ orally at ZT2 once per week and were placed in a new, clean cage. At the end of the experiments, histological analysis of brain lesions was performed with cresyl violet staining.

\section{Measuring of D-serine}

The CSF samples were mixed with twofold volumes of acetonitrile and centrifuged at 12000 r.p.m. for $10 \mathrm{~min}$ at $20^{\circ} \mathrm{C}$. The supernatant was mixed with $200 \mathrm{mM}$ sodium borate buffer ( $\mathrm{pH} 8.8$ ), and $5 \mathrm{mg} / \mathrm{ml}$ (S)-NIFE ((S)-N-(4nitrophenoxycarbonyl)-L-phenylalanine 2- methoxyethyl ester; Santa Cruz Biotechnology) in acetonitrile was then added. Then, HCL aqueous solution was added, and $5 \mathrm{ml}$ of the reaction mixture was subjected to the UPLC-MS/MS system. The quantitation method of $\mathrm{D} / \mathrm{L}$-amino acid is described previously (Visser et al, 2011) and used in this study with minor modifications. The derivatized amino acids were separated on an Acquity UPLC system (Waters, Milford, MA), and analyzed with a triple-quadrupole mass spectrometer, AB Sciex 5500QTRAP (AB Sciex, Framingham, $M A)$. The AB Sciex Analyst 1.6.2 software was used to control these instruments and to quantitate each amino acid.

\section{Data and Statistical Analyses}

The results are expressed as the mean \pm SEM. The comparisons of sleep/wake changes were analyzed using a two-way ANOVA followed by a Holm-Sidak test, when appropriate. The $\mathrm{Tb}$ values were expressed as an average for each 10-min period and were analyzed using a two-way repeated measure ANOVA followed by a Holm-Sidak test, when appropriate. The CBF values were converted into the percentage of perfusion units between pre- and postadministration (the average value $15 \mathrm{~min}$ before administration was $=100 \%$ ) and expressed as an average from 30 to $45 \mathrm{~min}$ after injection because the effects of the drugs were obvious at this range. The data were analyzed through oneway ANOVA followed by a Holm-Sidak test, when appropriate. All statistical analyses were conducted using SigmaStat software version 11.2 (Systat Software, Chicago, IL). In all cases, differences were considered significant at a level of $p<0.05$.

\section{RESULTS}

\section{Glycine Induces NREM Sleep Accompanied by a Decrease in Core Temperature}

The beneficial effects of glycine on subjective and objective sleep quality have been reported in subjects with insomniac tendencies (Inagawa et al, 2006; Yamadera et al, 2007). Therefore, in this sleep study, the rats were subjected to mild sleep disturbance in a new cage environment. To confirm whether a new cage environment induces acute sleep disturbance, the rats were transferred to a new, clean cage, and $\mathrm{Tb}$ and locomotor activity were compared with the values recorded when the rats were returned to the habituated home cage. Compared with the habituated home cage, the new cage environment significantly elevated $\mathrm{Tb}$ and locomotor activity during the first $2 \mathrm{~h}$ after cage exchange (Figure 1a and $\mathrm{b}$ ), consistent with previous reports (Cano et al, 2008; Oka et al, 2001). The rats orally received vehicle or glycine $(2 \mathrm{~g} / \mathrm{kg})$ at ZT2 with the cage 
exchange. Glycine treatment significantly decreased wakefulness compared with vehicle during the first $90 \mathrm{~min}$ after administration (sum for $90 \mathrm{~min}$ in vehicle, $72.8 \mathrm{~min} \pm 4.8$; glycine, $53.6 \mathrm{~min} \pm 2.5)$ and significantly increased NREM sleep (vehicle, $15.8 \mathrm{~min} \pm 4.1$; glycine, $35.0 \mathrm{~min} \pm 2.5$; Figure $1 \mathrm{c}$ and $\mathrm{d}$ ). Total episodes and mean duration of
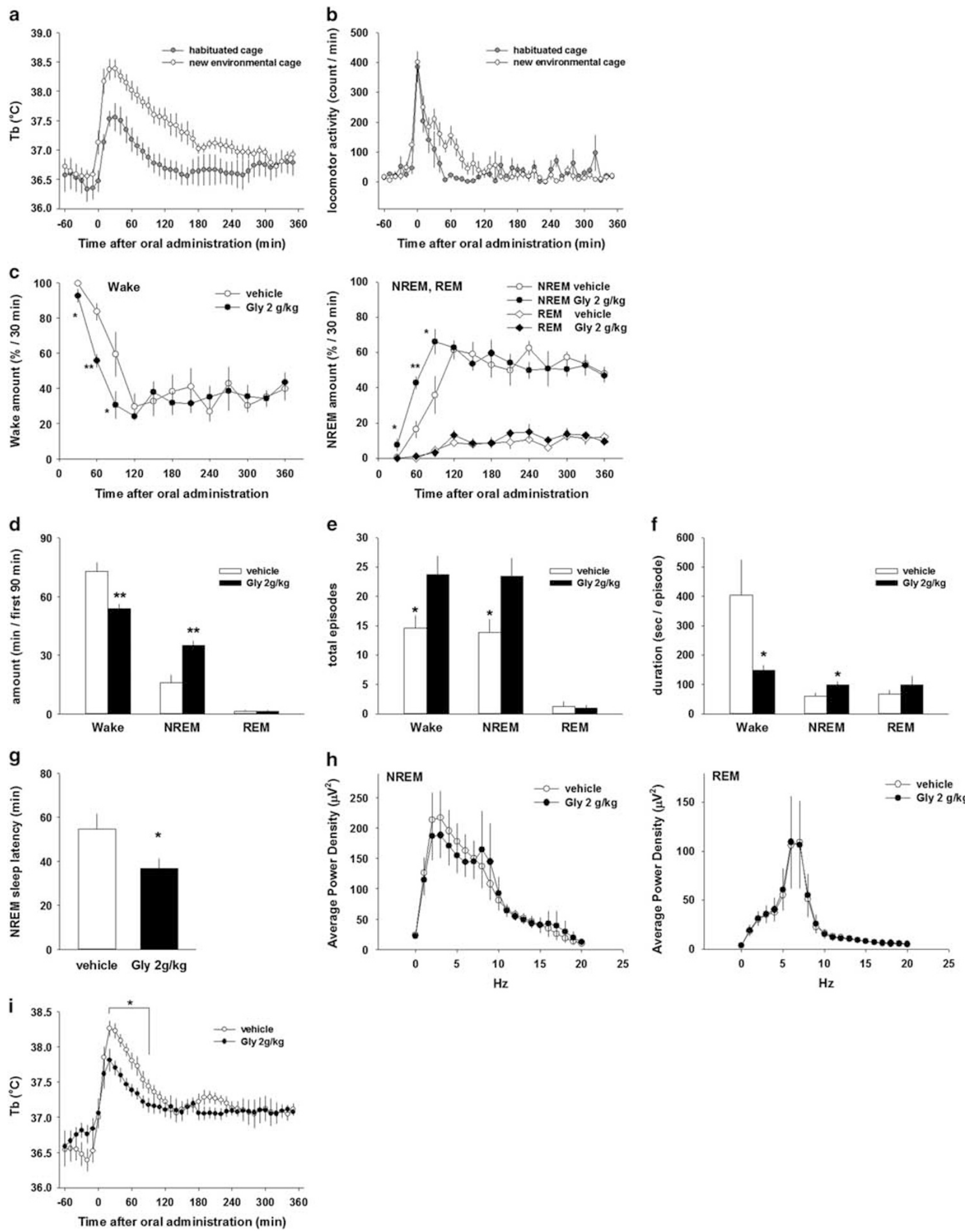
NREM sleep during the first $90 \mathrm{~min}$ also significantly increased with glycine treatment compared with vehicle (Figure 1e and $\mathrm{f}$ ). Total episodes of wakefulness during the first 90 min significantly increased, and the mean duration of wakefulness was significantly decreased with glycine treatment compared with vehicle (Figure $1 \mathrm{e}$ and $\mathrm{f}$ ). In addition, NREM sleep latency was significantly shortened by glycine administration (vehicle, $54.67 \pm 7.14 \mathrm{~min}$; glycine, $36.79 \pm 4.71 \mathrm{~min}$; Figure $1 \mathrm{~g}$ ). The reason for increasing wake episodes after glycine administration was not clear, but the experimental condition (ie, acute insomnia model) and effects on NREM sleep (ie, increase in NREM episode) may influence on the observed effects.

There was a moderate enhancement of theta power but no difference in EEG delta power in the first $2 \mathrm{~h}$ of NREM sleep (Figure 1h). Despite the accumulating results showing glycinergic regulation of REM sleep (Chase et al, 1989; Holstege et al, 1991; Morales et al, 2006; Soja et al, 1991), oral administration of glycine did not affect REM sleep in all cases. In accordance with the changes in sleep, oral administration of glycine significantly decreased $\mathrm{Tb}$ during the first 90 min after manipulation compared with vehicle (Figure 1i).

\section{NMDA, but not Glycine Receptors in the Brain Mediate Glycine-Induced Peripheral Heat Loss}

The negative correlation between sleep onset latency and peripheral heat loss via vasodilatation is a generally accepted model, as shown during natural sleep in humans (Krauchi et al, 1999, 2000; Krauchi and Wirz-Justice, 2001). Similarly in rats, $\mathrm{Tb}$ decreased, whereas tail-skin temperature increased at the initiation of NREM sleep (Alfoldi et al, 1990). We therefore examined the mechanisms that lower $\mathrm{Tb}$ following glycine administration and their relationship to sleep effects. A dose-dependent effect of glycine on $\mathrm{Tb}$ was observed even in the habituated cage (Figures $2 a$ and $b$ ). $\mathrm{Tb}$ is rigidly regulated by the integration of heat production in the body's core and heat loss from skin surfaces (Nakamura, 2011). To examine the thermoregulatory mechanism of exogenous glycine, laser-Doppler imaging was used to measure blood flow in the plantar paw surface. Oral administration of glycine under anesthesia significantly increased CBF at 30-45 min (Figure 2c), over a period during which the maximum decrease in $\mathrm{Tb}$ (Figure $2 \mathrm{~b}$ ) and the onset of sleep (NREM sleep latency, time from the injection to the first 1-min period of NREM sleep) was observed in the sleep study (Figure 1). The change occurred in a dose-dependent manner (vehicle, $97.0 \% \pm 3.8 ; 1 \mathrm{~g} / \mathrm{kg}$ glycine, $\quad 101.3 \% \pm 2.72 ; \quad 2 \mathrm{~g} / \mathrm{kg}$ glycine, $111.3 \% \pm 3.2$; Figure 2d).
Our previous whole-body autoradiography study showed that ingested glycine at a dose of $2 \mathrm{~g} / \mathrm{kg}$ passively diffuses across the blood-brain barrier through non-specific transportation and significantly elevates CSF glycine concentrations to a level higher than the $\mathrm{ED}_{50}$ of NMDA receptors and lower than that of glycine receptors (Kawai et al, 2011). We therefore performed ICV injection of glycine or D-serine, a modulator of the glycine site of NMDA receptors. ICV injection of glycine increased $\mathrm{CBF}$ in a dose-dependent manner. A higher dose of D-serine elevated CBF equivalent to $130 \mathrm{nmol}$ of glycine (D-serine, $116.4 \% \pm 3.7$; glycine, $114.6 \% \pm 4.3$; Figure 2e). Pretreatment with the NMDA receptor antagonists AP5 or CGP78608 significantly attenuated the effect of glycine (glycine, $110.3 \% \pm 3.0$; in the presence of AP5, 98.8\% \pm 3.2 ; CGP78608, $87.5 \% \pm 4.1$; Figure 2e). The glycine receptor antagonist strychnine $(30 \mathrm{nmol})$ did not inhibit the glycine $(130 \mathrm{nmol})$-induced increase in $\mathrm{CBF}$ (glycine, $114.6 \% \pm 4.3$; strychnine, $112.9 \% \pm 9.2$; Figure $2 \mathrm{e}$ ), suggesting that glycine induces peripheral vasodilatation via a glycine-binding site on NMDA receptors in the brain.

\section{Microinjection of Glycine into the SCN Increases CBF via NMDA Receptors}

It is well known that hypothalamic nuclei are involved in thermoregulation. The preoptic area, especially the MPO, functions as a thermoregulatory center by integrating afferent thermosensory signals and providing command signals to peripheral effectors through efferent neural and neuroendocrine pathways (Nakamura, 2011). On the other hand, the SCN is a crucial structure for the regulation of circadian rhythms in sleep and $\mathrm{Tb}$ (Saper et al, 2005). The MPO and SCN are therefore believed to be independently responsible for the homeostatic and circadian regulation of $\mathrm{Tb}$, respectively. We hypothesized that glycine activates hypothalamic areas through NMDA receptors and mediates sleep-promoting and hypothermic effects. To investigate this hypothesis, we examined c-Fos expression in the MPO, SCN, and periventricular/paraventricular nucleus of the hypothalamus. Because baseline c-Fos expression was high during the light period, and the light period was not suitable for evaluating c-Fos enhancement, the rats were orally administered glycine $(2 \mathrm{~g} / \mathrm{kg})$ or vehicle at ZT16 and killed in the dark $30 \mathrm{~min}$ after administration. We had confirmed that oral administration of glycine at 1 and $2 \mathrm{~g} / \mathrm{kg}$ at ZT 0,4 , 12 , 16 significantly reduced $\mathrm{Tb}$ (30 min after drug administration; Supplementary Figure 1). Glycine administration markedly increased the number of c-Fos positive neurons in the MPO (vehicle, $84 \pm 25$ cells $/ \mathrm{mm}^{2}$; glycine, $153 \pm 15$ cells/ $\mathrm{mm}^{2}$ ); however, there was no difference in c-Fos expression

Figure I The effects of glycine on core temperature and sleep in a new cage environment. Core body temperature (a) and locomotor activity (b) were recorded from 60 min before cage exchange. All values are the mean \pm SEM $(N=7)$. (c) Wake (left), NREM (middle), and REM (right) sleep amount changes are shown as the percentage of time spent in each stage every $30 \mathrm{~min}$ after oral administration of vehicle and $2 \mathrm{~g} / \mathrm{kg} g l y c i n e ~(N=7$ each). Amount (d), total episode number (e), and mean duration ( $f$ ) of each stage during the first 90 min after oral administration. Glycine treatment significantly increased NREM sleep compared with vehicle during the first $90 \mathrm{~min}$ after administration, but it had no effect on all parameters after 90 min. Asterisks indicate significant difference $(* p<0.05$, ** $p<0.0 \mathrm{I}$ ). (g) NREM sleep latency was defined as the time from oral administration to the first appearance of a NREM sleep episode lasting longer than I min. (h) The distribution of EEG power during NREM and REM sleep. The data include all artifact-free sleep epochs during the first $2 \mathrm{~h}$ after oral administration. (i) Glycine administration significantly decreased core body temperature compared with vehicle. 

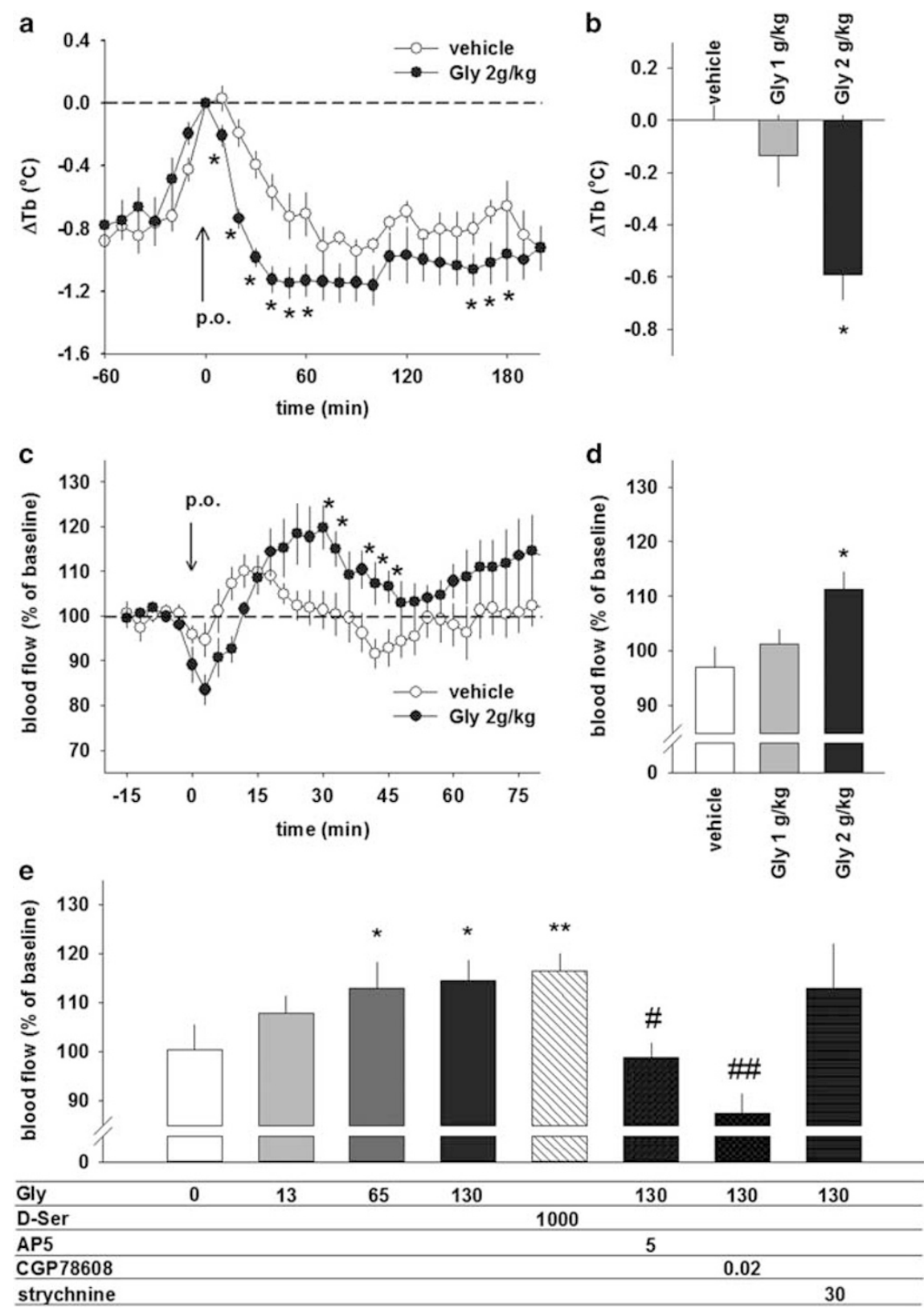

Figure 2 Dose-dependent decreases in core body temperature and increases in skin blood flow following peripheral and central administration of glycine. (a) The changes in rat core body temperature ( $\Delta$ core body temperature) were recorded from 60 min before administration. Each symbol represents the mean \pm SEM $(N=8)$. (b) The difference in $\Delta$ core body temperature 30 min after oral administration between the vehicle treatment group and the I- or 2 -g/kg glycine treatment group. The data are presented as the mean \pm SEM. $* p<0.05$ values are based on comparisons with the vehicle treatment group. (c) Changes in cutaneous blood flow (CBF) were recorded from 15 min before oral administration. Each symbol represents the mean \pm SEM ( $N=8-10)$. (d) The ratio of CBF 30-45 min after oral administration to 0-15 min before administration (baseline). (e) The effect of intracerebroventricular administration of glycine and glycine-related compounds on CBF. The data are presented as the mean \pm SEM $(N=5-10)$. $* p<0.05$ values are based on comparisons with baseline. $\# p<0.05, \# \# p<0.01$ values are based on comparisons with the absence of inhibitors of NMDA receptors or glycine receptors.

levels in the periventricular/paraventricular nucleus of the hypothalamus (vehicle, $147 \pm 41$ cells $/ \mathrm{mm}^{2}$; glycine, $163 \pm 35$ cells $/ \mathrm{mm}^{2}$ ). In the SCN, c-Fos expression significantly increased in the dorsomedial area (vehicle, $990 \pm 258$ cells $/ \mathrm{mm}^{2}$; glycine, $2541 \pm 554$ cells $/ \mathrm{mm}^{2}$ ) but not in the ventrolateral area (vehicle, $696 \pm 171$ cells $/ \mathrm{mm}^{2}$; glycine, $1294 \pm 316$ cells $/ \mathrm{mm}^{2}$; Figure 3 ).

The c-Fos immunohistochemistry results raised the possibility that exogenous glycine can directly activate hypothalamic areas that are functionally important for temperature control. To determine the primary action site of exogenous glycine, microinjection of glycine into the MPO, dSPZ, and SCN was performed in combination with laser-Doppler imaging. Bilateral injection into the SCN but not the MPO or the dSPZ significantly increased CBF equivalent to an ICV injection of $130 \mathrm{nmol}$ of glycine (MPO, 91.7\% $\pm 10.1 ;$ dSPZ, $91.0 \% \pm 11.8 ;$ SCN, $118.2 \% \pm 1.9$; ICV $114.6 \% \pm 4.3$; Figure $4 \mathrm{a})$, suggesting that the $\mathrm{SCN}$ is the 
a
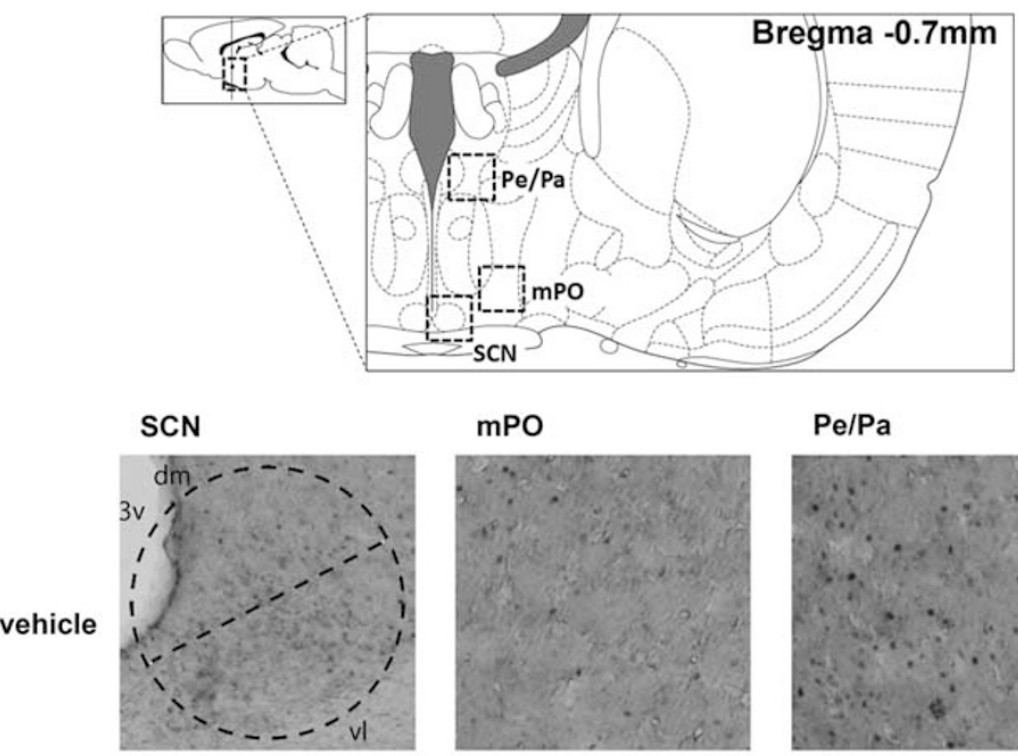

$$
\mathrm{mPO}
$$

$\mathrm{Pe} / \mathrm{Pa}$
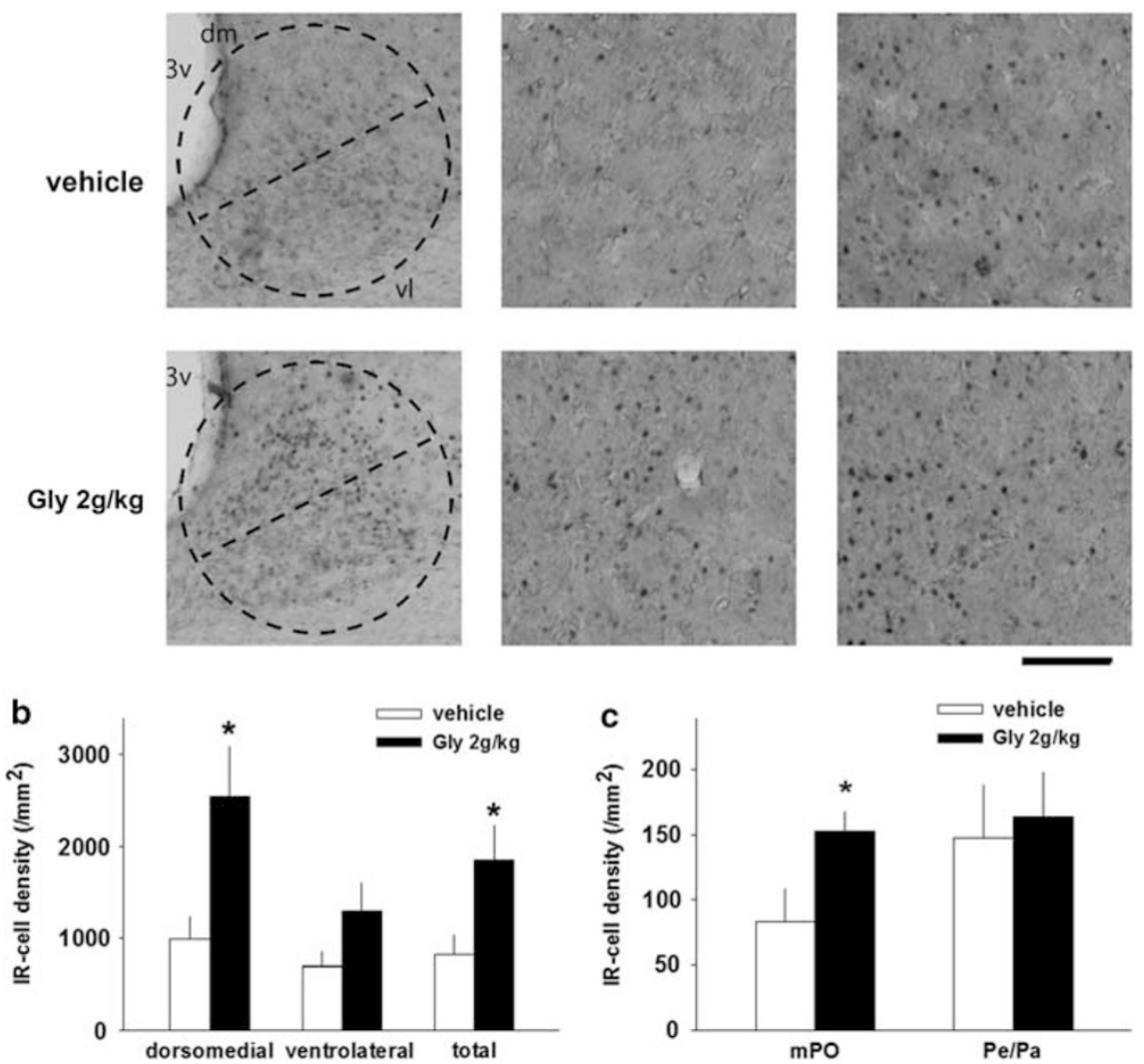

Figure 3 Oral administration of glycine induces c-Fos expression in the hypothalamic nuclei. (a) Typical images of c-Fos immunoreactive cells in each hypothalamic area. (b) The density of c-Fos immunoreactive cells in the SCN. (c) The density of c-Fos immunoreactive cells in Pe/Pa and mPO. The asterisks indicate a significant difference between the vehicle-treated group and the glycine-treated group $(* p<0.05)$.

primary target of glycine in the $\mathrm{CBF}$ increase. We further confirmed this result through the selective blockade of the glycine site on NMDA receptors in the SCN. The effect of glycine was dose dependent and antagonized by pretreatment with L-701324, a potent and selective antagonist of the glycine-binding site of NMDA receptors $(130 \mathrm{nmol}$ of glycine, $118.2 \% \pm 1.9$; in the presence of L-701324, $102.7 \% \pm 3.7$; Figure 4b). Likewise, CBF elevation by D-serine was blocked by L-701324 (1000 nmol of D-serine, $122.8 \% \pm 5.4$; in the presence of L-701324, 103.8\% \pm 3.2 ). L-serine, which is an isomer of D-serine with reduced ability to activate NMDA receptors (Kleckner and Dingledine, 1988), had less effect on CBF than glycine and D-serine. Moreover, we measured D-serine concentration in CSF after oral administration of $2 \mathrm{~g} / \mathrm{kg}$ of glycine (Figure 5). The level of D-serine did not significantly increase in CSF after $30 \mathrm{~min}$ (vehicle, $1.79 \mu \mathrm{M} \pm 0.04 ; 2 \mathrm{~g} / \mathrm{kg}$ glycine, $1.92 \mu \mathrm{M} \pm 0.14$ ), $2 \mathrm{~h}$ (vehicle, $1.82 \mu \mathrm{M} \pm 0.18 ; 2 \mathrm{~g} / \mathrm{kg}$ glycine, $1.95 \mu \mathrm{M} \pm 0.16$ ) and $8 \mathrm{~h}$ (vehicle, $2.25 \mu \mathrm{M} \pm 0.15 ; 2 \mathrm{~g} / \mathrm{kg}$ glycine, $1.89 \mu \mathrm{M} \pm 0.08$ ) after oral administration of glycine, supporting the minimum influence of $\mathrm{D}$-serine by oral administration of glycine. Taken together, our results strongly suggest that glycine acts primarily on NMDA receptors in the SCN and causes vasodilatation.

\section{Effects of Glycine on both Core Temperature and Sleep are Abolished in SCN-Lesioned Rats}

Although a series of microinjection experiments identified the SCN as the primary target of glycine in the brain for peripheral vasodilatation, it remains unclear whether glycine acts directly on thermoeffector organs such as 

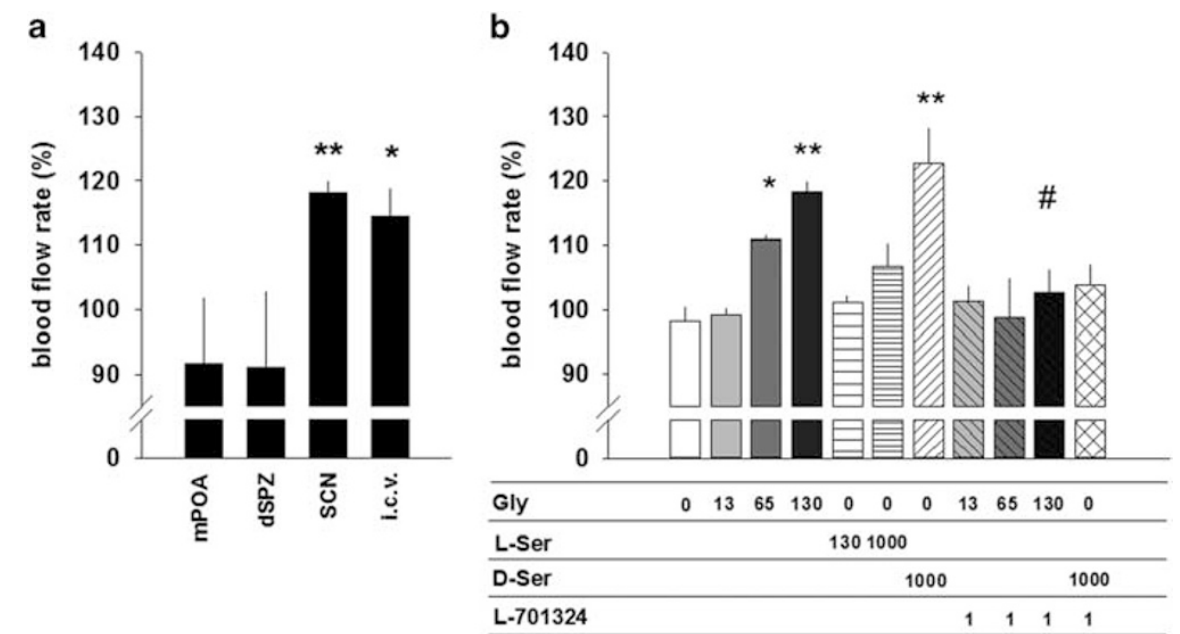

Figure 4 The microinjection of glycine into the thermoregulatory areas in the presence or absence of the specific antagonist of NMDA receptors. (a) The bilateral injection of glycine into the thermoregulatory areas. (b) The bilateral injection of glycine into the suprachiasmatic nucleus in the presence or absence of L-70 I 324, a specific antagonist of NMDA receptors. Each column represents the mean \pm SEM $(n=4-6)$. The asterisks indicate a significant difference between CBF 0-15 min before glycine treatment and CBF 30-45 min after glycine treatment $(* p<0.05, * * p<0.01)$. \# indicates a significant difference between the presence and absence of L-70I324. (\#p<0.05).

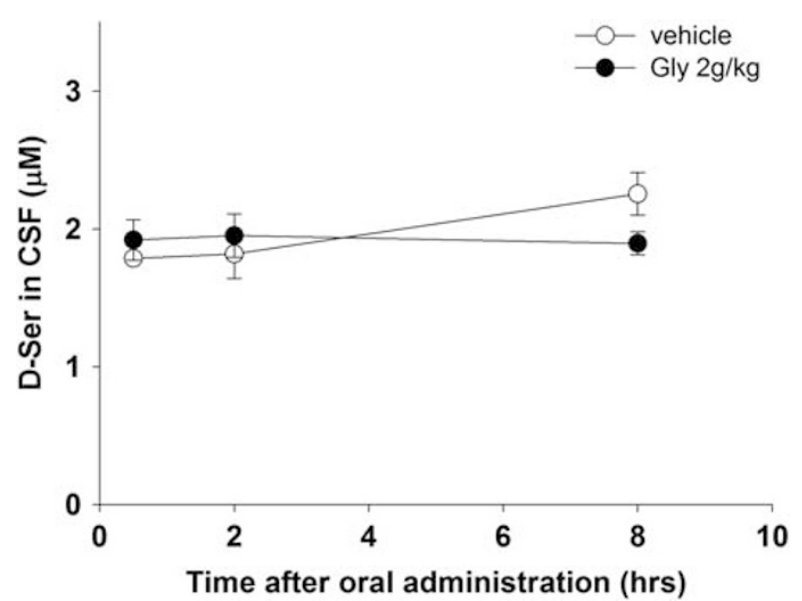

Figure 5 The concentration of D-serine in CSF after oral administration of glycine. Concentration-time curves of D-serine in CSF following oral administration of vehicle (empty circles) or $2 \mathrm{~g} / \mathrm{kg}$ of glycine (filled circles). Each symbol represents the mean $\pm \operatorname{SEM}(n=5)$.

blood vessel muscles to induce heat loss and whether the SCN is also essential for the sleep-promoting effects of glycine. To directly address these issues, we performed sleep recording following glycine administration in SCN-lesioned $(\mathrm{SCNx})$ rats. Eleven out of fourteen rats that received bilateral radio frequency lesions of the SCN showed loss of circadian rhythms in $\mathrm{Tb}$ during the constant dark condition, whereas sham-operated rats exhibited a stable freerunning rhythm (Figure 6a). After recovery from EEG/EMG surgery, SCNx and sham groups orally received vehicle or glycine $(2 \mathrm{~g} / \mathrm{kg})$ with mild sleep disturbance. The hypothermic effect of glycine remained intact in the sham group, whereas SCN lesions abolished the effect (Figure 6b). In addition to the hypothermic effect, glycine failed to affect sleep amount, number of episodes, and mean duration of NREM sleep in the SCNx group (Figure 6c-e). The glycine- induced short NREM sleep latency observed in the sham group disappeared in the SCNx group (Figure 6f), suggesting that the SCN is a critical structure required for the thermoregulatory and sleep-promoting effects of glycine.

\section{DISCUSSION}

Despite evidence from human trials that glycine intake before bedtime improves sleep quality both subjectively and objectively (Bannai et al, 2012; Inagawa et al, 2006; Yamadera et al, 2007), details of its mechanism remain poorly understood. We have revealed the site of action of glycine that promotes sleep and a novel mechanism for regulating sleep and temperature. In acute sleep disturbance during the resting period, glycine administration promoted sleep and shortened NREM sleep latency associated with a decrease in $\mathrm{Tb}$ (Figure 1). The time course of the decrease in $\mathrm{Tb}$ correlated well with the timing of sleep onset and the rise in CBF (Figure $1 \mathrm{~g}$ and Figure 2a-d). Peripheral vasodilatation was mediated by activation of NMDA but not glycine receptors in the brain (Figure $2 \mathrm{e}$ and $\mathrm{f}$ ). This finding is consistent with the pharmacokinetics and distribution in the brain following glycine administration (Kawai et al, 2011). Glycine administration increases L-serine (Kawai et al, 2011) and L-serine can be converted to D-serine by serine racemase. To exclude the possibility that the effects observed may partially be mediated by $\mathrm{D}$-serine, we investigated D-serine concentrations in CSF $30 \mathrm{~min}, 2$ and $8 \mathrm{~h}$ after the oral administration of $2 \mathrm{~g} / \mathrm{kg}$ glycine. We found no significant change in the CSF D-serine level (Figure 5). This result indicates that glycine itself acts on NMDA receptors. Among the hypothalamic nuclei responsible for thermoregulation, the SCN was determined to be the primary action site of glycine (Figures 3 and 4 ). Because the MPO is influenced by the SCN directly (Watts et al, 1987) or indirectly via projections from the dSPZ (Lu et al, 2001), MPO activation following glycine administration is most likely to be a secondary effect that is involved in 
a
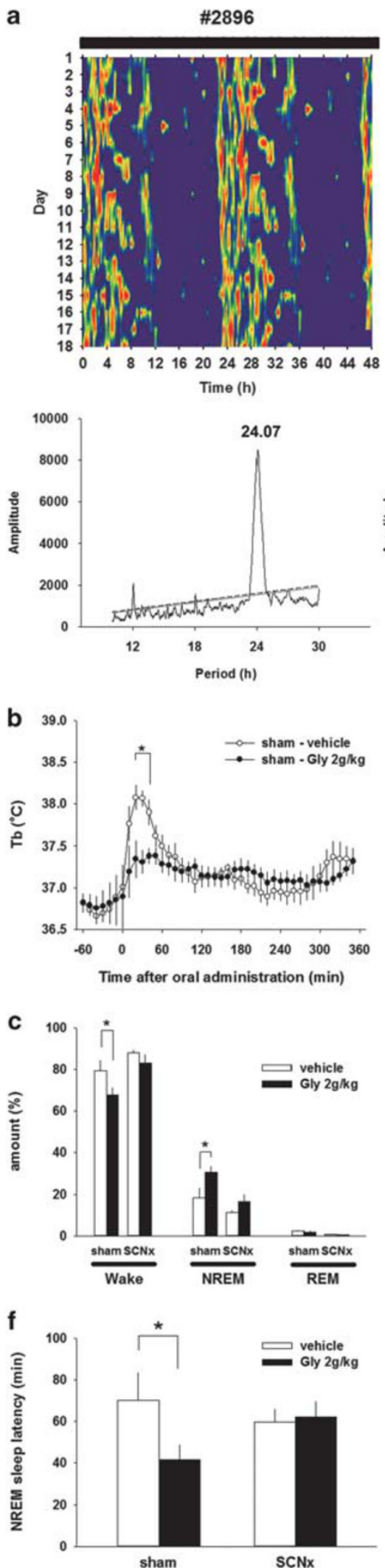

\#2883
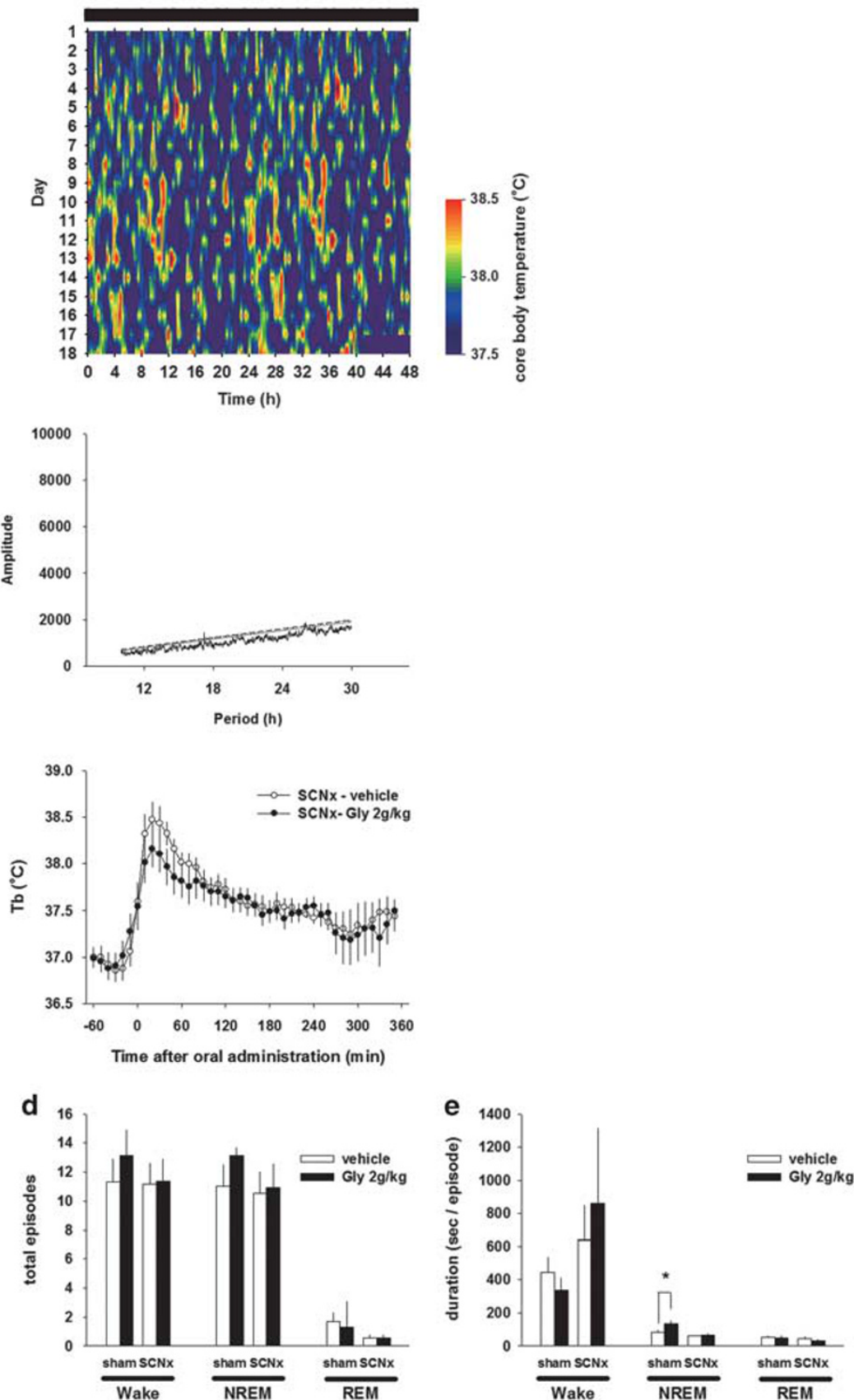

Figure 6 The evaluation of the effects of glycine on sleep in SCNx rats. (a) Representative temperature records (upper) and $\chi^{2}$ periodograms (lower) of sham (left) and SCNx (right) rats. Circadian tau was given for significant peaks $(p<0.00 \mathrm{I}$ ). (b) The core body temperature was recorded from 60 min before oral administration in sham (left) and SCNx (right) rats. All values are the mean \pm SEM ( $n=6$ for sham rats, $n=1 \mid$ for SCNx rats). The asterisks indicate significant differences $(* p<0.05)$. Amount (c), total episode number (d), and mean duration (e) of each stage in sham and SCNx rats during the first 90 min after oral administration. (f) NREM sleep latency was not shortened by glycine administration in SCNx rats. 
peripheral vasodilatation. The result that the sleep-promoting and hypothermic effects of glycine were abolished in the SCNx supports our hypothesis (Figure 6).

We demonstrated that glycine significantly increased the activity of SCN neurons by c-Fos immunostaining during the active period (Figure 3 ) and significantly reduced $\mathrm{Tb}$ both at day and night (Supplementary Figure 1). As the c-fos expression in the SCN was maximally high during daytime in rats, we were unable to demonstrate by c-fos experiments that glycine administration further enhances the activity of the SCN neurons during the resting period. We therefore conducted pharmacological study of neurotransmission of glycine via NMDA receptors (Figure 4) and investigated physiological evidence of loss of Tb control by SCN lesions (Figure 6). All of these results support our hypothesis that increasing SCN activity by glycine mediates drop of $\mathrm{Tb}$ and the improvement in sleep during the resting phase of rats.

In view of energy conservation, a mutual interaction between sleep and thermoregulation is reasonable. Numerous neuroanatomical and electrophysiological studies have demonstrated that sleep and temperature are predominantly regulated by the VLPO and MPO, respectively, under the influence of the SCN circadian rhythm (Saper et al, 2005). To initiate and maintain sleep, VLPO efferent axons directly inhibit arousal-related monoaminergic neurons through GABAergic and galaninergic projections. On the other hand, to lower temperature, the inhibitory circuit descending from the MPO is enhanced to suppress spinal motor outputs, resulting in skin vasodilatation and reduced thermogenesis (Nakamura, 2011). In addition, MPO neurons with sleep-related discharge have projections to monoaminergic neurons (Alam et al, 1995; Steininger et al, 2001), implying an intimate link between sleep and temperature.

Although we elucidated the mechanism of action of exogenous glycine, these findings shed light on the potential role of endogenous glycine in sleep and thermoregulation. A substantial number of glycine-immunoreactive fibers project to several structures of the hypothalamus (Rampon et al, 1996; van den Pol and Gorcs, 1988). Despite a weak glycinergic innervation of the SCN, organotypic slice cultures of rat SCN showed a robust circadian rhythm in intrinsic release of glycine with 10 times higher levels than glutamate and aspartate (Shinohara et al, 1998). In addition, several lines of evidence support the idea that glycine has a dual role as an excitatory modulator and an inhibitory neurotransmitter through NMDA and glycine receptors on SCN neurons and modulates circadian phase shifting (Harrington et al, 1999; Ito et al, 1991; Kallingal and Mintz, 2010; Mordel et al, 2011). Considering that light entrains the circadian oscillation by enhancing NMDA receptor activity in direct glutamatergic retinal inputs to the SCN (Colwell, 2001; Pennartz et al, 2001), endogenous glycine likely modulates sensitivity to glutamate to synchronize circadian rhythms and influence phase resetting of the clock. Exogenous glycine may augment this modulatory action. In addition to its role in circadian organization, glycine seems to be closely related to the neuropeptide arginine vasopressin (AVP). AVP is secreted from the SCN shell in autocrine and paracrine manners during the light phase, in synchrony with glycine release (Kalsbeek et al,
1995; Shinohara et al, 1998). We have reported that AVP mRNA expression increases 4.2 -fold with glycine administration during the light but not the dark period (Bannai et al, 2012). Central infusion of AVP in nanogram quantities also reduces psychological stress-induced hyperthermia resulting from restrain stress (Terlouw et al, 1996). Therefore, AVP may be partly involved in the decrease in Tb following glycine administration; however, the details of its mechanism remain to be elucidated.

A recent study using mice proposed different mechanisms of glycine action. Hondo et al (2011) reported that orexin neurons receive glycinergic innervations and that an intraperitoneal injection of $2 \mathrm{~g} / \mathrm{kg}$ glycine increases NREM sleep in the dark but not the light period by suppressing orexin neurons through strychnine-sensitive glycine receptors, in contrast with our results. Thus, we performed sleep analysis using the same protocol as Hondo et al (2011) and observed that intraperitoneal injection of glycine caused severe hypothermia $\left(<33^{\circ} \mathrm{C}\right)$ for $5 \mathrm{~h}$, continuous muscle atonia accompanied with intermittent shivering and an abnormal EEG pattern (sporadic delta activity on a low amplitude mixed frequency EEG background) that does not occur during physiological sleep during both the light and dark periods in not only wild-type but also in orexin/ataxin3 transgenic mice, in which the orexin neurons degenerate (Hara et al, 2001; data not shown). These observations might be caused by acute glycine toxicity. The dose used for ip injection was close to a lethal dose (LD), as the oral $\mathrm{LD}_{50}$ for mice has been reported to be $4.92 \mathrm{~g} / \mathrm{kg}$. By contrast, the glycine dose we used for the rat experiments was approximately $1 / 4$ of the $\mathrm{LD}_{50}$ for rats $(\mathrm{ie}, 7.93 \mathrm{~g} / \mathrm{kg}$ ). In the present study, we note that no rats exhibited these abnormal EEG and behavioral changes, although a moderate enhancement of theta waves during sleep was observed through FFT analysis.

However, in vitro experiments clearly demonstrated that glycine hyperpolarized orexin neurons (Hondo et al, 2011). Furthermore, in light of the glycinergic innervations and dual responses to glycine observed in electrophysiological studies (Hondo et al, 2011; Karnani et al, 2011a,2011b), it is possible that orexin neurons have physiological glycinergic regulation, and further studies are required.

In summary, we conclude that exogenous glycine promotes sleep by modulating thermoregulation and circadian rhythms through the activation of NMDA receptors in the SCN. Studies are required to further elucidate the mechanisms by which glycine orchestrates SCN function with the proper timing and magnitude, which will provide a better understanding of the physiological role of glycine and its therapeutic potential for improving sleep quality.

\section{FUNDING AND DISCLOSURE}

N.S., M.O., and S.N. are funded by Ajinomoto Co., Inc. N.K., S.K., Y.T., and T.T. are employees of Ajinomoto Co., Inc.

\section{ACKNOWLEDGEMENTS}

We thank Dr Yoichi Ueta and Dr Takashi Maruyama (University of Occupational and Environmental Health, Kitakyusyu, Japan) for their valuable comments. 


\section{REFERENCES}

Alam MN, McGinty D, Szymusiak R (1995). Neuronal discharge of preoptic/anterior hypothalamic thermosensitive neurons: relation to NREM sleep. Am J Physiol 269(5 Pt 2): R1240-R1249.

Alfoldi P, Rubicsek G, Cserni G, Obal F Jr. (1990). Brain and core temperatures and peripheral vasomotion during sleep and wakefulness at various ambient temperatures in the rat. Pflugers Arch 417: 336-341.

Bannai M, Kawai N, Ono K, Nakahara K, Murakami N (2012). The effects of glycine on subjective daytime performance in partially sleep-restricted healthy volunteers. Front Neurol 3: 61.

Barrett J, Lack L, Morris M (1993). The sleep-evoked decrease of body temperature. Sleep 16: 93-99.

Campbell SS, Broughton RJ (1994). Rapid decline in body temperature before sleep: fluffing the physiological pillow? Chronobiol Int 11: 126-131.

Cano G, Mochizuki T, Saper CB (2008). Neural circuitry of stressinduced insomnia in rats. J Neurosci 28: 10167-10184.

Chase MH, Soja PJ, Morales FR (1989). Evidence that glycine mediates the postsynaptic potentials that inhibit lumbar motoneurons during the atonia of active sleep. J Neurosci 9: 743-751.

Colwell CS (2001). NMDA-evoked calcium transients and currents in the suprachiasmatic nucleus: gating by the circadian system. Eur J Neurosci 13: 1420-1428.

Coyle JT, Tsai G (2004). The NMDA receptor glycine modulatory site: a therapeutic target for improving cognition and reducing negative symptoms in schizophrenia. Psychopharmacology 174: 32-38.

Curtis DR, Hosli L, Johnston GA (1968). A pharmacological study of the depression of spinal neurones by glycine and related amino acids. Exp Brain Res 6: 1-18.

Czeisler CA, Weitzman E, Moore-Ede MC, Zimmerman JC, Knauer RS (1980). Human sleep: its duration and organization depend on its circadian phase. Science 210: 1264-1267.

Dijk DJ, von Schantz M (2005). Timing and consolidation of human sleep, wakefulness, and performance by a symphony of oscillators. J Biol Rhythms 20: 279-290.

Elliot EE, White JM (2001). The acute effects of zolpidem compared to diazepam and lorazepam using radiotelemetry. Neuropharmacology 40: 717-721.

Fujiki N, Cheng T, Yoshino F, Nishino S (2009). Specificity of direct transition from wake to REM sleep in orexin/ataxin-3 transgenic narcoleptic mice. Exp Neurol 217: 46-54.

Hara J, Beuckmann CT, Nambu T, Willie JT, Chemelli RM, Sinton CM et al (2001). Genetic ablation of orexin neurons in mice results in narcolepsy, hypophagia, and obesity. Neuron 30: 345-354.

Harrington ME, Hoque S, Hall A, Golombek D, Biello S (1999). Pituitary adenylate cyclase activating peptide phase shifts circadian rhythms in a manner similar to light. J Neurosci 19: 6637-6642.

Heresco-Levy U, Javitt DC, Ermilov M, Mordel C, Silipo G, Lichtenstein M (1999). Efficacy of high-dose glycine in the treatment of enduring negative symptoms of schizophrenia. Arch Gen Psychiat 56: 29-36.

Holstege JC, Bongers CM (1991). A glycinergic projection from the ventromedial lower brainstem to spinal motoneurons. An ultrastructural double labeling study in rat. Brain Res 566: 308-315.

Hondo M, Furutani N, Yamasaki M, Watanabe M, Sakurai T (2011). Orexin neurons receive glycinergic innervations. PLoS One 6: e25076.

Inagawa $\mathrm{K}$, Hiraoka $\mathrm{T}$, Kohda $\mathrm{T}$, Yamadera $\mathrm{W}$, Takahashi $\mathrm{M}$ (2006). Subjective effects of glycine ingestion before bedtime on sleep quality. Sleep Biol Rhythms 4: 75-77.

Ito C, Wakamori M, Akaike N (1991). Dual effect of glycine on isolated rat suprachiasmatic neurons. Am J Physiol 260(2 Pt 1): C213-C218.
Johnson JM, Taylor WF, Shepherd AP, Park MK (1984). LaserDoppler measurement of skin blood flow: comparison with plethysmography. J Appl Physiol 56: 798-803.

Johnson JW, Ascher P (1987). Glycine potentiates the NMDA response in cultured mouse brain neurons. Nature 325: 529-531.

Kallingal GJ, Mintz EM (2010). An NMDA antagonist inhibits light but not GRP-induced phase shifts when administered after the phase-shifting stimulus. Brain Res 1353: 106-112.

Kalsbeek A, Buijs RM, Engelmann M, Wotjak CT, Landgraf R (1995). In vivo measurement of a diurnal variation in vasopressin release in the rat suprachiasmatic nucleus. Brain Res 682: 75-82.

Karnani MM, Apergis-Schoute J, Adamantidis A, Jensen LT, de Lecea L, Fugger L et al (2011a). Activation of central orexin/ hypocretin neurons by dietary amino acids. Neuron 72: 616-629.

Karnani MM, Venner A, Jensen LT, Fugger L, Burdakov D (2011b). Direct and indirect control of orexin/hypocretin neurons by glycine receptors. J Physiol 589(Pt 3): 639-651.

Kawai N, Bannai M, Seki S, Koizumi T, Shinkai K, Nagao K et al (2011). Pharmacokinetics and cerebral distribution of glycine administered to rats. Amino Acids 42: 2129-2137.

Kleckner NW, Dingledine R (1988). Requirement for glycine in activation of NMDA-receptors expressed in Xenopus oocytes. Science 241: 835-837.

Krauchi K, Cajochen C, Werth E, Wirz-Justice A (1999). Warm feet promote the rapid onset of sleep. Nature 401: 36-37.

Krauchi K, Cajochen C, Werth E, Wirz-Justice A (2000). Functional link between distal vasodilation and sleep-onset latency? Am J Physiol Regul Integr Comp Physiol 278: R741-R748.

Krauchi K, Wirz-Justice A (2001). Circadian clues to sleep onset mechanisms. Neuropsychopharmacology 25(5 Suppl): S92-S96.

Lavie P (1997). Melatonin: role in gating nocturnal rise in sleep propensity. J Biol Rhythms 12: 657-665.

Lu J, Zhang YH, Chou TC, Gaus SE, Elmquist JK, Shiromani P et al (2001). Contrasting effects of ibotenate lesions of the paraventricular nucleus and subparaventricular zone on sleep-wake cycle and temperature regulation. J Neurosci 21: 4864-4874.

Markwald RR, Lee-Chiong TL, Burke TM, Snider JA, Wright KP Jr. (2010). Effects of the melatonin MT-1/MT-2 agonist ramelteon on daytime body temperature and sleep. Sleep 33: 825-831.

Morales FR, Sampogna S, Rampon C, Luppi PH, Chase MH (2006). Brainstem glycinergic neurons and their activation during active (rapid eye movement) sleep in the cat. Neuroscience 142: 37-47.

Mordel J, Karnas D, Inyushkin A, Challet E, Pevet P, Meissl H (2011). Activation of glycine receptor phase-shifts the circadian rhythm in neuronal activity in the mouse suprachiasmatic nucleus. J Physiol 589(Pt 9): 2287-2300.

Nagao K, Bannai M, Kawai N, Endo T (2007). Glycine decreases core body temperature in healthy volunteer.. Jap Soc Sleep Res The 32nd Annual Meeting of Japanese Society of Sleep Research: 1-S-017.

Nakamura K (2011). Central circuitries for body temperature regulation and fever. Am J Physiol Regul Integr Comp Physiol 301: R1207-R1228.

Oka T, Oka K, Hori T (2001). Mechanisms and mediators of psychological stress-induced rise in core temperature. Psychosom Med 63: 476-486.

Pennartz CM, Hamstra R, Geurtsen AM (2001). Enhanced NMDA receptor activity in retinal inputs to the rat suprachiasmatic nucleus during the subjective night. J Physiol 532(Pt 1): 181-194.

Peterson SL (1994). Diazepam potentiation by glycine in pentylenetetrazol seizures is antagonized by 7-chlorokynurenic acid. Pharmacol Biochem Behav 47: 241-246.

Rampon C, Luppi PH, Fort P, Peyron C, Jouvet M (1996). Distribution of glycine-immunoreactive cell bodies and fibers in the rat brain. Neuroscience 75: 737-755.

Reeds PJ (2000). Dispensable and indispensable amino acids for humans. J Nutr 130: 1835S-1840S. 
Sakurai T, Mieda M, Tsujino N (2010). The orexin system: roles in sleep/wake regulation. Ann NY Acad Sci 1200: 149-161.

Saper CB, Lu J, Chou TC, Gooley J (2005). The hypothalamic integrator for circadian rhythms. Trends Neurosci 28: 152-157.

Shinohara K, Honma S, Katsuno Y, Abe H, Honma K (1998). Circadian release of amino acids in the suprachiasmatic nucleus in vitro. Neuroreport 9: 137-140.

Soja PJ, Lopezrodriguez F, Morales FR, Chase MH (1991). The postsynaptic inhibitory control of lumbar motoneurons during the atonia of active sleep-effect of strychnine on motoneuron properties. J Neurosci 11: 2804-2811.

Steininger TL, Gong H, McGinty D, Szymusiak R (2001). Subregional organization of preoptic area/anterior hypothalamic projections to arousal-related monoaminergic cell groups. J Comp Neurol 429: 638-653.

Stone BM, Turner C, Mills SL, Nicholson AN (2000). Hypnotic activity of melatonin. Sleep 23: 663-669.

Sumova A, Travnickova Z, Mikkelsen JD, Illnerova H (1998). Spontaneous rhythm in c-Fos immunoreactivity in the dorsomedial part of the rat suprachiasmatic nucleus. Brain Res $\mathbf{8 0 1}$ : 254-258.

Swanson LW (2004). Brain maps: structure of the rat brain. A Laboratory Guide with Printed and Electronic Templates for Data, Models and Schematics. 3rd edn. Elsevier: Amsterdam.

Terlouw EM, Kent S, Cremona S, Dantzer R (1996). Effect of intracerebroventricular administration of vasopressin on stressinduced hyperthermia in rats. Physiol Behav 60: 417-424.
Toth E, Lajtha A (1986). Antagonism of phencyclidine-induced hyperactivity by glycine in mice. Neurochem Res 11: 393-400.

van den Pol AN, Gorcs T (1988). Glycine and glycine receptor immunoreactivity in brain and spinal cord. J Neurosci 8: 472492.

Visser WF, Verhoeven-Duif NM, Ophoff R, Bakker S, Klomp LW, Berger $\mathrm{R}$ et al (2011). A sensitive and simple ultra-highperformance-liquid chromatography-tandem mass spectrometry based method for the quantification of D-amino acids in body fluids. J Chromatogr A 1218: 7130-7136.

Watts AG, Swanson LW, Sanchez-Watts G (1987). Efferent projections of the suprachiasmatic nucleus: I. Studies using anterograde transport of Phaseolus vulgaris leucoagglutinin in the rat. J Comp Neurol 258: 204-229.

Werman R, Davidoff RA, Aprison MH (1968). Inhibitory of glycine on spinal neurons in the cat. J Neurophysiol 31: 81-95.

Yamadera W, Inagawa $\mathrm{K}$, Chiba S, Bannai $\mathrm{M}$, Takahashi $\mathrm{M}$, Nakayama K (2007). Glycine ingestion improves subjective sleep quality in human volunteers, correlating with polysomnographic changes. Sleep Biol Rhythms 5: 126-131.

Zhdanova IV, Wurtman RJ, Lynch HJ, Ives JR, Dollins AB, Morabito C et al (1995). Sleep-inducing effects of low doses of melatonin ingested in the evening. Clin Pharmacol Ther 57: 552-558.

Zulley J, Wever R, Aschoff J (1981). The dependence of onset and duration of sleep on th circadian rhythm of rectal temperature. Pflugers Arch 391: 314-318.

Supplementary Information accompanies the paper on the Neuropsychopharmacology website (http://www.nature.com/npp) 